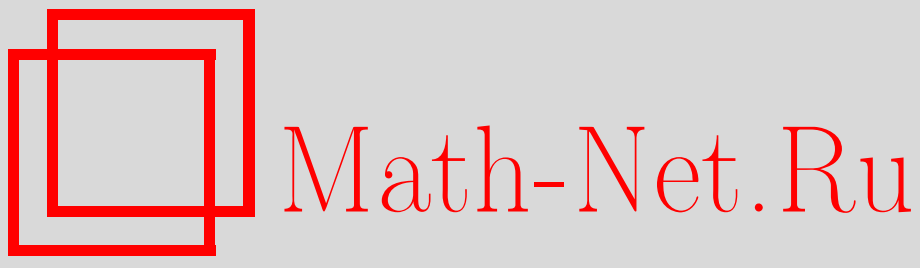

Ю. И. Ингстер, О проверке гипотезы, близкой к простой, Теория вероятн. и ее примен., 2000, том 45, выпуск 2, 356368

DOI: https://doi.org/10.4213/tvp468

Использование Общероссийского математического портала Math-Net.Ru подразумевает, что вы прочитали и согласны с пользовательским соглашением http://www.mathnet.ru/rus/agreement

Параметры загрузки:

IP: 35.173 .137 .237

26 апреля 2023 г., 18:30:49

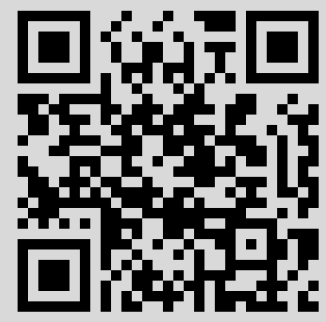


6. Борисов И.С., Боровков А.А. Аппроксимация второго порядка случайных ломаных в принципе инвариантности Донскера-Прохорова. - Теория вероятн. и ее примен., 1986, т. XXXI, в. 2, с. 225-245.

7. Мосваия B.E. Асимптотическое представление для процесса отношения правдоподобия в случае разрынной плотности. - Сиб. матем. журн., 1994, т. 35, № 2, c. $416-423$.

8. Borisov I.S. Strong Poisson and mixed approximations of sums of independent random variables in Banach spaces. - Siberian Adv. Math., 1993, v. 3, № 2, p. 1-13.

9. Пинелис И.Ф., Сахамеяхо А.И. Замечания о неравенствах для вероятностей больших уклонений. - Теория вероятн. и ее примен., 1985, т. XXX, в. 1, с. 127131.

10. Саханенхо А. И. О точности нормальной аппроксимации в принципе инвариантности. - Асимптотический анализ распределений случайных процессов. Новосибирск: Наука, 1989, с. 40-66.

11. Komlós J., Major P., Tusnády G. An approximation of partial sums of independent RV's and the sample DF. - Z. Wahrscheinlichkeitstheor. verw. Geb., 1976, B. 34, H. 1, S. 33-58.

12. Боровков A. A. Теория вероятностей. М.: Наука, 1976, 352 с.

13. Добруиия P.Л. Задание системы случайных величин при помоши условных распределений. - Теория вероятн. и ее примен., 1970, т. XV, в. 3, с. 469-497.

Поступила в редакцию 23.V.1997

(c) $2000 \mathrm{r}$.

ИНГСТЕР Ю. И.*

\section{О ПРОВЕРКЕ ГИПОТЕЗЫ, БЛИЗКОЙ К ПРОСТОЙ ${ }^{1)}$}

Пусть наблюдается $n$-мерный гауссовский вектор $x=v+\xi$, где $v \in \mathbf{R}^{n}$ - неизвестный вектор средних, $\xi$ - стандартный $n$-мерный гауссовский вектор. При $n \rightarrow \infty$ рассматривается асимптотически минимаксная задача проверки гипотезы $H_{0}:\|v\|_{p} \leqslant R_{n, 0}$ против альтернативы $H_{1}:\|v\|_{p} \geqslant R_{n, 1}$. Известно [3], что при простой гипотезе $H_{0}$ (т.е. при $R_{n, 0}=0$ ) условия минимаксной различимости и неразличимости имеют вид $R_{n, 1} / R_{n, 1, p}^{*} \rightarrow \infty, R_{n, 1} / R_{n, 1, p}^{*} \rightarrow 0$ соответственно и выражаются в терминах «критических радиусов» $R_{n, 1, p}^{*}$. Нас интересует вопрос: сколь малым может быть $R_{n, 0}$ для сохранения этих условий различимости и неразличимости?

Ответ имеет вид $R_{n, 0}=o\left(R_{n, 0, p}^{*}\right)$ и выражается в терминах «критических радиусов» $R_{n, 0, p}^{*}$, вид которых зависит от четности $p$. В частности, показатель стелени «критических радиусов» $R_{n, 0, p}^{*}$ как функция от $p$ имеет разрыв слева при четных $p>2$; кроме того, $R_{n, 0, p}^{*} \asymp R_{n, 1, p}^{*}$, лишь если $p$ четно. Эти результаты переносятся на модель, соответствуюшую наблюдениям неизвестного сигнала $f$ из соболевского или бесовского класса в гауссовском белом шуме.

Аналогичные эффекты в задаче оценивания функционала $\Phi(f)=\|f\|_{p}$ были недавно установлены в работе [12].

Ключевые слова и фразы: минимаксная проверка гипотез, непараметрические гипотезы и альтернативы, соболевские и бесовские классы.

* Петербургский государственный университет путей сообщения, Московский пр., 9, 190031 С.-Петербург, Россия.

1) Работа выполнена при поддержке Российского фонда фундаментальных исследований (проекты 96-01-00684, 98-01-04108) и гранта ведуцих научных школ (проект 96-15-96199). 


\section{1. Введение}

1.1. Постановка задачи. Мы рассмотрим два варианта задачи проверки гипотез.

1. Проверка гипотез о среднем гауссовского вехтора возрастакией размерности. Пусть наблюдается последовательность $n$-мерных гауссовских векторов $x=v+\xi, x=\left(x_{1}, \ldots, x_{n}\right)$, где $v=\left(v_{1}, \ldots, v_{n}\right) \in \mathbf{R}^{n}$ - неизвестный вектор средних, a $\xi=\left(\xi_{1}, \ldots, \xi_{n}\right)-$ стандартный $n$-мерный гауссовский вектор, т.е. случайные величины $\xi_{1}, \ldots, \xi_{n}$ независимы и $\xi_{i} \sim \mathcal{N}(0,1)$. Рассмотрим задачу проверки гипотезы $H_{0}: v \in V_{n, 0}$ против альтернативы $H_{1}: v \in V_{n, 1}$, где

$$
V_{n, 0}=\left\{v \in \mathbf{R}^{n}:\|v\|_{p} \leqslant R_{n, 0}\right\}, \quad V_{n, 1}=\left\{v \in \mathbf{R}^{n}:\|v\|_{p} \geqslant R_{n, 1}\right\} .
$$

Здесь $\|v\|_{p}=\left(\sum_{i}\left|v_{i}\right|^{p}\right)^{1 / p}, 0<p<\infty, 0 \leqslant R_{n, 0}<R_{n, 1}$ - заданные последовательности. Нас будут интересовать условия минимаксной различимости и неразличимости в этой задаче при $n \rightarrow \infty$. Именно, для любого теста $\psi=\psi_{n}(x)$ (под тестом понимается измеримая функция на $\mathbf{R}^{n}$ со значениями в интервале $\left.[0,1]\right)$ рассмотрим вероятности ошибок I и II рода $\alpha_{n}(\psi, v)=\mathbf{E}_{n, v}(\psi), \beta_{n}(\psi, v)=\mathbf{E}_{n, v}(1-\psi)$, их макскмальные значения

$$
\alpha_{n}\left(\psi, V_{n, 0}\right)=\sup _{v \in V_{n}, 0} \alpha_{n}(\psi, v), \quad \beta_{n}\left(\psi, V_{n, 1}\right)=\sup _{v \in V_{n, 1}} \beta_{n}(\psi, v)
$$

и сумму $\gamma_{n}\left(\psi, V_{n, 0}, V_{n, 1}\right)=\alpha_{n}\left(\psi, V_{n, 0}\right)+\beta_{n}\left(\psi_{n}, V_{n, 1}\right)$. Здесь и ниже $\mathbf{E}_{n, v}-$ среднее по гауссовской мере $\mathbf{P}_{n, v}$ на $\left(\mathbf{R}^{n}, \mathscr{B}^{n}\right)$ со средним $v$ и единичной матрицей ховариаций. Положим

$$
\gamma_{n}=\gamma_{n}\left(V_{n, 0}, V_{n, 1}\right)=\inf _{\psi} \gamma_{n}\left(\psi, V_{n, 0}, V_{n, 1}\right) ; \quad 0 \leqslant \gamma_{n}\left(V_{n, 0}, V_{n, 1}\right) \leqslant 1
$$

Будем говорить, что гипотеза $H_{0}$ и альтернатива $H_{1}$ состоятельно различимь, если $\gamma_{n} \rightarrow 0$, и асимптотически жеразличимы, если $\gamma_{n} \rightarrow 1 ;$ последовательность тестов $\psi_{n}$ будем называть состоятельной (в минкмаксном смысле), если $\gamma_{n}\left(\psi_{n}, V_{n, 0}, V_{n, 1}\right) \rightarrow 0$. Здесь и ниже для рассматриваемой задачи, если не оговорено противное, пределы рассматривакотся при $n \rightarrow \infty$.

2. Проверка аипотез о сигкале в гауссовском белом шуме. Пусть для семейства $\varepsilon>0, \varepsilon \rightarrow 0$, наблюдается семейство реализаций $X_{\varepsilon}=\left\{X_{\varepsilon}(t), t \in(0,1)\right\}$ случайного процесса $d X_{\varepsilon}(t)=f(t) d t+\varepsilon d \omega(t)(\omega(t)-$ стандартный винеровский процесс), соответствующего наблюдению сигнала $f=f(t), t \in(0,1), f \in L_{2}(0,1)$, в гауссовском белом шуме интенсивности $\varepsilon$. Нулевая гипотеза и альтернатива имеют вид $H_{0}: f \in F_{\varepsilon, 0}, H_{1}: f \in F_{\varepsilon, 1}$, где

$$
\begin{aligned}
& F_{e, 0}=\left\{f \in L_{2}(0,1):\|f\|_{p} \leqslant \rho_{\varepsilon, 0}\right\} \\
& F_{\varepsilon, 1}=\left\{f \in L_{2}(0,1):\|f\|_{\sigma, p} \leqslant C,\|f\|_{p} \geqslant \rho_{\varepsilon, 1}\right\} .
\end{aligned}
$$

Здесь $\|f\|_{p}$ - норма в $L_{p}(0,1),\|\cdot\|_{\sigma, p}$ - норма Соболева, Никольского или Бесова в пространстве функций гладкости $\sigma>0 ; 1 \leqslant p<\infty$ - параметр, определяющий $L_{p}$-норму для измерения гладкости, $C>0$ - постоянная, $0 \leqslant \rho_{\varepsilon, 0}<\rho_{\varepsilon, 1}$ - заданные числовые семейства.

Мы рассмотрим случай нормы Бесова, $\|\cdot\|_{\sigma, p}=\|\cdot\|_{\sigma, p, h}$, где (мы приводим одно из определений нормы Бесова; см. также [7], [8], [10], [11]):

$$
\begin{aligned}
\|f\|_{\sigma, p, h} & =\|f\|_{p}+\left(\int_{0}^{1}\left(\frac{w_{r, p}(f, u)}{u^{\sigma}}\right)^{q} \frac{d u}{u}\right)^{1 / h} ; \\
\|f\|_{\sigma, p, \infty} & =\|f\|_{p}+\sup _{0<u<1} \frac{w_{r, p}(f, u)}{u^{\sigma}} .
\end{aligned}
$$

Здесь $\|f\|_{p}$ - норма в $L_{p}(0,1) ; w_{r, p}(f, u)=\left\|\Delta_{u}^{(r)} f\right\|_{L_{p}[0,1-r u]}, u>0$, есть $L_{p}$-норма $r$-й разности функции $f$ :

$$
\left(\Delta_{u}^{(r)} f\right)(t)=\sum_{k=0}^{r} C_{r}^{k}(-1)^{k} f(t+k u), \quad 0<t+r u<1,
$$

где $r>\sigma+1$ и $r \geqslant \sigma+2$ при целом $\sigma$. 
Поскольку сформулированные ниже результаты не зависят от параметра $h$, аналогичные результаты для шаров Соболева и Никольского вытекают из известных теорем вложения [11].

Нас будут интересовать условия мннимаксной различимости $\left(\gamma_{\epsilon} \rightarrow 0\right)$ и неразличимости $\left(\gamma_{\varepsilon} \rightarrow 1\right)$ в этой задаче при $\varepsilon \rightarrow 0$ (определения аналогичны задаче $1 \mathrm{c}$ заменой мер $\mathbf{P}_{n, v}$ на меры $\mathbf{P}_{\varepsilon, f}$, соответствуюшие случайному процессу $X_{\varepsilon}$ ).

Для простой нулевой гипотезы $\left(\rho_{\varepsilon, 0}=0\right)$ задачи типа 2 (и их модификации для пространства последовательностей $l_{2}$; шары в квазинорме $\|\cdot\|_{\sigma, p}$ соответствуют $l_{p}$-эллипсоидам) изучались в работах [1]-[3]; в работах [4]-[9] изучался также неоднородный случай (норма $\|\cdot\|_{\sigma, p}$ заменяется на норму $\|\cdot\|_{\sigma, q}$ ). Там же изучались модификации этих задач для пространства $l_{2}$; при этом шары в норме $\|\cdot\|_{\sigma, q}$ (или квазинорме при $0<q<1$ ) соответствуют $l_{q}$-эллипсоидам или телам Бесова.

Для однородного случая $p=q$, который интересует нас в этой работе, при простой нулевой гипотезе условия различимости и неразличимости в задаче 2 имеют вид (см. [3, теорема 2.5]):

$$
\gamma_{\varepsilon} \rightarrow 0 \Longleftrightarrow \frac{\rho_{\varepsilon, 1}}{\rho_{\varepsilon, 1}^{*}} \rightarrow \infty ; \quad \gamma_{\varepsilon} \rightarrow 1 \Longleftrightarrow \frac{\rho_{\varepsilon, 1}}{\rho_{\varepsilon, 1}^{*}} \rightarrow 0
$$

здесь критические радиусы $\rho_{\varepsilon, 1}^{*}$ имеют вид

$$
\rho_{e, 1}^{*}=\rho_{e, 1, p}^{*}= \begin{cases}\varepsilon^{4 \sigma /(4 \sigma+1)} & \text { при } p \leqslant 2 \\ \varepsilon^{2 \sigma /(2 \sigma+1-1 / p)} & \text { при } p>2 .\end{cases}
$$

По существу, эти результаты выводятся из соответствующих результатов для задачи 1 (см. [3, пा. $4.2,4.3 ; 5.2,5.3])$ :

$$
\gamma_{n} \rightarrow 0 \Longleftrightarrow \frac{R_{n, 1}}{R_{n, 1}^{*}} \rightarrow \infty ; \quad \gamma_{n} \rightarrow 1 \Longleftrightarrow \frac{R_{n, 1}}{R_{n, 1}^{*}} \rightarrow 0,
$$

где критинеские радиусы $R_{n, 1}^{*}$ имеют вид

$$
R_{n, 1}^{*}=R_{n, 1, p}^{*}= \begin{cases}n^{1 / p-1 / 4} & \text { при } p \leqslant 2 \\ n^{1 / 2 p} & \text { при } p>2 .\end{cases}
$$

Нас будет интересовать следующий вопрос: для каких $R_{n, 0}>0$ uли $\rho_{\varepsilon, 0}>0$ сохракяются условия различимости и неразличимости вида (1.1), (1.2) в задаче 2 или вида (1.3), (1.4) в задаче 1 ?

1.2. Основные результаты. Ответ на этот вопрос формулируется в терминах критических радиусов $R_{n, 0}^{*}$ или $\rho_{\varepsilon, 0}^{*}$, вид которых зависит от четности $p$. Положим

$$
\begin{aligned}
R_{n, 0}^{*}=R_{n, 0, p}^{*} & = \begin{cases}n^{1 / 2 p} & \text { при } p \leqslant 2, \\
n^{1 / p-1 / 4 k} & \text { при } 2 k \leqslant p<2(k+1), k=1,2, \ldots ;\end{cases} \\
\rho_{\varepsilon, 0}^{*} & =\rho_{\varepsilon, 0, p}^{*}= \begin{cases}\varepsilon^{(4 \sigma-1+2 / p) /(4 \sigma+1)} & \text { при } p \leqslant 2, \\
\varepsilon^{(2 \sigma+1 / 2 k-1 / p) /(2 \sigma+1-1 / p)} & \text { при } 2 k \leqslant p<2(k+1) .\end{cases}
\end{aligned}
$$

Следуюшая теорема показывает, что условие $R_{n, 0} / R_{n, 0}^{*} \rightarrow 0$ необходимо и достаточно для справедливости условия различимости и неразличимости вида (1.3), (1.4) в задаче 1.

Теорема 1.1. 1) Нижние границы. Пусть $R_{n, 0} / R_{n, 0}^{*} \rightarrow \infty$ в задаче 1. Тогда суиествует такая последовательность $R_{n, 1}$, ито $R_{n, 1} / R_{n, 1}^{*} \rightarrow \infty u \gamma_{n} \rightarrow 1$.

2) Верхние границы. Пусть $R_{n, 0} / R_{n, 0}^{*} \rightarrow 0$ в эадаче 1 . Тогда $\gamma_{n} \rightarrow 0$ для любоц последовательности $R_{n, 1}$ такой, ито $R_{n, 1} / R_{n, 1}^{*} \rightarrow \infty$.

Этот результат переносится и на задачу 2.

Теорема 1.2. 1) Нижние границы. Пусть $\rho_{\varepsilon, 0} / \rho_{\varepsilon, 0}^{*} \rightarrow \infty$ в задаче 2. Тогда cyuествует maxoе семейство $\rho_{\varepsilon, 1}$, ито $\rho_{\varepsilon, 1} / \rho_{\varepsilon, 1}^{*} \rightarrow \infty u \gamma_{\varepsilon} \rightarrow 1$.

2) Верхние границы. Пусть $\rho_{\varepsilon, 0} / \rho_{\varepsilon, 0}^{*} \rightarrow 0$ в задаче 2. Тогда $\gamma_{\varepsilon} \rightarrow 0$ для мюбого семейства $\rho_{\varepsilon, 1}$ такого, ито $\rho_{\varepsilon, 1} / \rho_{\varepsilon, 1}^{*} \rightarrow \infty$. 
Отметим, что $R_{n, 0}^{*} \asymp R_{n, 1}^{*}$ и $\rho_{\varepsilon, 0}^{*} \asymp \rho_{\varepsilon, 1}^{*}$, лишь если $p$ четно; в противном случае $R_{n, 0}^{*}=o\left(R_{n, 1}^{*}\right)$ и $\rho_{\varepsilon, 0}^{*}=o\left(\rho_{\varepsilon, 1}^{*}\right)$. Кроме того, показатели степени $n$ и $\varepsilon$ в определениях критических радиусов $R_{n, 0}^{*}$ и $\rho_{\varepsilon, 0}^{*}$ как функции от $p$ имеют разрыв слева при четных $p>2$.

Аналогичные эффекты в задаче оценивания функционала $\Phi(f)=\|f\|_{p}$, где $f$ есть неизвестный сигнал из липшицевского класса, наблюдаемый в гауссовском белом шуме, были недавно установлены в работе [12]. Именно, в [12] показано, что порядки точности минимаксного оценивания $\|f\|_{p}$ существенно различаются при $p=2 k$ и при $p \neq 2 k$.

Доказательство п. 1) теоремы 1.1 будет дано в разделе 2, а п. 2) теоремы 1.1 в разделе 3; там же мы опишем структуру состоятельных в минимаксном смысле тестов при выполнении условий различимости. В разделе 4 мы перенесем результаты на задачу 2.

\section{2. Нижние границы в задаче 1}

2.1. Метод построения нижних границ. Построение нижних границ основано на асимптотическом варианте байесовского подхода и следует схеме, описанной в $\left[3\right.$, пп. 2.1, 4.1] для случая простой гипотезы $H_{0}$ (см. также [5], [9]). Чтобы получить соотношение $\gamma_{n} \rightarrow 1$, достаточно построить такие последовательности вероятностных мер $\pi_{n}^{(0)}, \pi_{n}^{(1)}$ на $\left(\mathbf{R}^{n}, \mathscr{B}^{n}\right)$, что выполнены следующие условия:

$$
\begin{gathered}
\text { (i) } \pi_{n}^{(0)}\left(V_{n, 0}\right) \rightarrow 1 ; \quad \text { (ii) } \pi_{n}^{(1)}\left(V_{n, 1}\right) \rightarrow 1 ; \\
\text { (iii) } \operatorname{var}\left(\mathbf{P}_{\pi_{n}^{(0)}}, \mathbf{P}_{\pi_{n}^{(1)}}\right) \rightarrow 0
\end{gathered}
$$

где $\mathbf{P}_{\pi_{n}^{(l)}}$ - смеси по мерам $\pi_{n}^{(l)}, l=0,1$ :

$$
\mathbf{P}_{\pi_{n}^{(l)}}(A)=\int \mathbf{P}_{n, v}(A) \pi_{n}^{(l)}(d v)
$$

$\mathbf{h}$

$$
\operatorname{var}\left(\mathbf{P}_{\pi_{n}^{(0)}}, \mathbf{P}_{\pi_{n}^{(1)}}\right)=\mathbf{E}_{\mathbf{P}_{n, 0}}\left|f_{\pi_{n}^{(1)}}(x)-f_{\pi_{n}^{(0)}}(x)\right|
$$

- расстояние по вариации ( $L_{1}$-расстояние) между смесями; здесь

$$
f_{\pi_{n}^{(l)}}(x)=\frac{d \mathbf{P}_{\pi_{n}^{(l)}}}{d \mathbf{P}_{n, 0}}(x)=\int_{\mathbf{R}^{n}} \exp \left(-\frac{\|v\|^{2}}{2}+(x, v)\right) \pi_{n}^{(l)}(d v) .
$$

Мы будем искать меры $\pi_{n}^{(l)}$ как произведения одинаховых одномерных мер $\pi_{n, l}$ на $\mathbf{R}^{1}: \pi_{n}^{(l)}\left(d v_{1} \cdots d v_{n}\right)=\pi_{n, l}\left(d v_{1}\right) \times \cdots \times \pi_{n, l}\left(d v_{n}\right)$. При этом

$$
f_{\pi_{n}^{(l)}}(x)=\prod_{i=1}^{n} f_{\pi_{n, l}}\left(x_{i}\right), \quad f_{\pi_{n, l}}(t)=\frac{d \mathbf{P}_{\pi_{n, l}}}{d \mathbf{P}_{1,0}}(t)=\int_{\mathbf{R}^{1}} \exp \left(-\frac{u^{2}}{2}+t u\right) \pi_{n, l}(d u),
$$

здесь $d \mathbf{P}_{\pi_{n, l}}$ - «одномерная» смесь по мере $\pi_{n, l}, f_{\pi_{n, l}}$ - плотности смеси относительно стандартной гауссовской меры $\mathbf{P}_{1,0}, l=0,1$. Используя неравенство Чебышева, легко видеть, что для проверки условий (i) и (ii) в (2.1) достаточно установить соотношения: для некоторых $C_{1}>1>C_{0}>0$

$$
\begin{array}{ll}
n \mathbf{E}_{\pi_{n, 0}}|t|^{p} \leqslant\left(C_{0} R_{n, 0}\right)^{p} ; & n \mathbf{D}_{\pi_{n, 0}}|t|^{p}=o\left(R_{n, 0}^{2 p}\right) ; \\
n \mathbf{E}_{\pi_{n, 1}}|t|^{p} \geqslant\left(C_{1} R_{n, 1}\right)^{p} ; & n \mathbf{D}_{\pi_{n, 1}}|t|^{p}=o\left(R_{n, 1}^{2 p}\right),
\end{array}
$$

где $\mathbf{D}_{\boldsymbol{\pi}}$ - дисперсия по мере $\boldsymbol{\pi}$.

3 а м е ч а и и е 2.1. Если выполнены соотношения (2.2), (2.3), причем в первых неравенствах имеет место равенство, то меры $\pi_{n}^{(l)}$ с вероятностью, стремящейся к 1 , сосредоточены внутри колец $\left\{1-\delta \leqslant\|v\|_{p} / C_{l} R_{n, l} \leqslant 1+\delta\right\}$ для любого $\delta>0$, $l=0,1$. 
Для заряда $r$ на $\left(\mathbf{R}^{1}, \mathscr{B}^{1}\right)$ обозначим

$$
\|r\|^{2}=\int_{\mathbf{R}^{1}} \int_{\mathbf{R}^{1}}\left(e^{u v}-1\right) r(d u) r(d v) .
$$

По поводу полунормы $\|r\|$ на пространстве зарядов см. [5], [9]; для наших целей мы можем считать заряды конечными и финитными.

Для проверки условия (iii) в (2.1) удобно следующее утверждение.

Лемма 2.1. Пусть при достаточно больиих $n$ для нехоторого $\delta>0$

$$
\inf _{t \in \mathbf{R}^{1}} f_{\pi_{n, l}}(t) \geqslant \delta, \quad l=0 \text { usu } 1 .
$$

Тогда для выполнения условия (iii) в (2.1) достаточяо, чтобы

$$
n\left\|\pi_{n, 1}-\pi_{n, 0}\right\|^{2}=n \mathbf{E}_{\mathbf{P}_{1,0}}\left(f_{\pi_{n, 1}}(t)-f_{\pi_{n, 0}}(t)\right)^{2}=o(1) .
$$

Доказательств о. В силу симметрии условий можно считать $l=0$. Используя неравенство $1+x \leqslant e^{x}$, видим, что

$$
\begin{aligned}
& \operatorname{var}^{2}\left(\mathbf{P}_{\pi_{n}^{(0)}}, \mathbf{P}_{\pi_{n}^{(1)}}\right) \leqslant \mathbf{E}_{\mathbf{F}_{n}^{(0)}}\left(\frac{f_{\pi_{n}^{(1)}}(x)}{f_{\pi_{n}^{(0)}}(x)}-1\right)^{2}=\mathbf{E}_{\mathbf{P}_{\pi_{n}}^{(0)}}\left(\frac{f_{\pi_{n}^{(1)}}(x)}{f_{\pi_{n}^{(0)}}(x)}\right)^{2}-1 \\
& =\left(\mathbf{E}_{\mathbf{P}_{\pi_{n}, 0}}\left(\frac{f_{\pi_{n, 1}}(t)}{f_{\pi_{n, 0}}(t)}-1\right)^{2}+1\right)^{n}-1 \leqslant \exp \left(n \mathbf{E}_{\mathbf{P}_{\pi_{n}, 0}}\left(\frac{f_{\pi_{n, 1}}(t)}{f_{\pi_{n, 0}}(t)}-1\right)^{2}\right)-1,
\end{aligned}
$$

так что для выполнения условия (iii) в (2.1) достаточно, чтобы

$$
n \mathbf{E}_{\mathbf{P}_{\pi_{n}, 0}}\left(\frac{f_{\pi_{n, 1}}(t)}{f_{\pi_{n, 0}}(t)}-1\right)^{2}=n \mathbf{E}_{\mathbf{P}_{1,0}} \frac{\left(f_{\pi_{n, 1}}(t)-f_{\pi_{n, 0}}(t)\right)^{2}}{f_{\pi_{n}, 0}(t)}=o(1) .
$$

Но при выполнении условия (2.5) соотношение (2.7) вытекает из (2.6). Лемма доказана.

Разлагая подынтегральную функцию (2.4) в ряд по степеням иv, получаем представление:

$$
\|r\|^{2}=\sum_{j=1}^{\infty} \frac{\left(\mu_{j}(r)\right)^{2}}{j !} ; \quad \mu_{j}(r)=\int_{\mathbf{R}^{1}} u^{j} r(d u)
$$

$\mu_{j}(r)$ - моменты заряда $r$, и если меры $\pi_{n, l}, l=0,1$, симметричны (только такие меры мы будем использовать ниже), то (2.6) можно представить в виде:

$$
n \sum_{j=1}^{\infty} \frac{\left(\mu_{2 j}\left(\pi_{n, 1}\right)-\mu_{2 j}\left(\pi_{n, 0}\right)\right)^{2}}{(2 j) !}=o(1)
$$

Схема построения мер $\pi_{n, l}, l=0,1$, ниже состоит в том, чтобы при выполнении условий $(2.2),(2.3)$ попытаться обратить в 0 как можно больше первых членов ряда (2.8).

Для меры $\pi$ на $\left(\mathbf{R}^{1}, \mathscr{B}^{1}\right)$ обозначим через $|\pi|=\inf \{T: \pi([-T, T])=1\}$ половину длины симметричного носителя меры. Удобно использовать следуюшее простое утверждение.

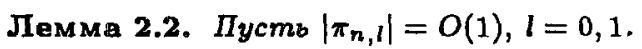

1) Пусть меры $\pi_{n, l}, l=0,1$, симметричкы. Тогда выполкеко (2.5).

2) Пусть выполнены первые условия (2.2), (2.3), причем в них имеет место равенство по порядку, и $R_{n, l} \rightarrow \infty, l=0,1$. Тогда выполнены и вторые услоous (2.2), (2.3).

3) Пусть меры $\pi_{n, l}, l=0,1$, симметричяы, $\mu_{2 j}\left(\pi_{n, 1}\right)=\mu_{2 j}\left(\pi_{n, 0}\right), j \leqslant k, k \geqslant 1, u$ $n\left(\mu_{2(k+1)}\left(\pi_{n, l}\right)\right)^{2}=o(1), l=0,1$. Тогда выполнено $(2.8)$. 
Д о к а з а т е л ь с т в о. Утверждение 1) вытекает из следующих оценок: для симметричной вероятностной меры $\pi$

$$
\begin{aligned}
f_{\pi}(t) & =\int_{\mathbf{R}^{1}} e^{-u^{2} / 2+u t} \pi(d u)=\frac{1}{2}\left(\int_{\mathbf{R}^{1}} e^{-u^{2} / 2+u t} \pi(d u)+\int_{\mathbf{R}^{1}} e^{-u^{2} / 2-u t} \pi(d u)\right) \\
& =\int_{\mathbf{R}^{1}} e^{-u^{2} / 2} \operatorname{ch}(t u) \pi(d u) \geqslant \int_{\mathbf{R}^{1}} e^{-u^{2} / 2} \pi(d u) \geqslant e^{-|\pi|^{2} / 2} .
\end{aligned}
$$

Утверждения 2) и 3) вытекают из неравенств $\mathbf{E}_{\pi}|t|^{a+b} \leqslant|\pi|^{a} \mathbf{E}_{x}|t|^{b}$ при $a>0, b>0$, $\mathbf{D}_{\pi}|t|^{p} \leqslant \mathbf{E}_{\pi}|t|^{2 p}$ и очевидных оценок для суммы ряда в $(2.8)$.

2.2. Случай $p \leqslant 2$. Проиллюстрируем эту схему для случая $p \leqslant 2$.

Пусть $R_{n, 0}=U_{n}^{1 / p} R_{n, 0}^{*}, U_{n} \rightarrow \infty$, причем можно считать, что $U_{n}=o\left(n^{a}\right)$ для достаточно малого $a>0$. Положим

$$
\pi_{n, 1}=\frac{\delta_{-b_{1}}+\delta_{b_{1}}}{2} ; \quad \pi_{n, 0}=(1-h) \delta_{0}+\frac{h\left(\delta_{-b_{0}}+\delta_{b_{0}}\right)}{2} ;
$$

здесь и ниже $\delta_{t}$ есть масса, сосредоточенная в точке $t \in \mathbf{R}^{1}$, так что меры симметричны и $\left|\pi_{n, l}\right|=b_{l}, l=0,1$. Пусть $b_{l}=b_{l, n}=o(1) ; h=h_{n}=o(1)$ - положительные последовательности, которые выбираются из условий:

$$
\begin{aligned}
& b_{1}^{2}=h b_{0}^{2} ; \quad h=n^{-1 / 2} A_{n}, \\
& 2 A_{n} b_{0}^{p}=U_{n}, \quad A_{n} b_{0}^{2} \rightarrow \infty, \quad A_{n} b_{0}^{2}=o\left(n^{1 / 4}\right), \quad A_{n} b_{0}^{4} \rightarrow 0
\end{aligned}
$$

(можно положить $2 A_{n}=U_{n}^{1+p / 2}, b_{0}=U_{n}^{-1 / 2}, a=\frac{1}{4}$ ). При этом выполнены условия (2.2), (2.3) для $R_{n, 1}=(n / 2)^{1 / p} b_{1}=2^{-1 / p} A_{n}^{1 / 2} b_{0} R_{n, 1}^{*} \gg R_{n, 1}^{*}$ :

$$
n \mathbf{E}_{\pi_{n, 0}}|t|^{p}=n h b_{0}^{p}=\frac{R_{n, 0}^{p}}{2} ; \quad n \mathbf{E}_{\pi_{n, 1}}|t|^{p}=n b_{1}^{p}=2 R_{n, 1}^{p} .
$$

Первое условие (2.9) позволяет аннулировать разность вторых моментов в (2.8), а для четвертых моментов имее:

$$
n\left(\mu_{4}\left(\pi_{n, 0}\right)\right)^{2}=n h^{2} b_{0}^{8}=A_{n}^{2} b_{0}^{8}=o(1) ; \quad n\left(\mu_{4}\left(\pi_{n, 1}\right)\right)^{2}=n b_{1}^{8}=n^{-1}\left(A_{n} b_{0}^{2}\right)^{4}=o(1) .
$$

С учетом леммы 2.2 п. 1) теоремы 1.1 при $p \leqslant 2$ доказан.

2.3. Случай $p \geqslant 2$. Пусть $2 k \leqslant p<2(k+1), k=1,2, \ldots$ Для последовательностей числовых наборов $z_{1}>z_{2}>\cdots>z_{k+1}>0, h_{1}, h_{2}, \ldots, h_{k+1} ; z_{i}=z_{i, n}$, $h_{i}=h_{i, n}>0,1 \leqslant i \leqslant k+1$, таких, что $H=\sum_{i} h_{i} \leqslant 1$, (их вид будет уточнен ниже) положим

$$
\begin{gathered}
H_{1}=\sum_{i: 1 \leqslant 2 i-1 \leqslant k+1} h_{2 i-1}, \quad H_{0}=\sum_{i: 2 \leqslant 2 i \leqslant k+1} h_{2 i} ; \quad r(z, h)=\frac{h\left(\delta_{-z}+\delta_{z}\right)}{2}, \\
\pi_{n, 1}=\left(1-H_{1}\right) \delta_{0}+\sum_{i: 1 \leqslant 2 i-1 \leqslant k+1} r\left(z_{2 i-1}, h_{2 i-1}\right) \\
\pi_{n, 0}=\left(1-H_{0}\right) \delta_{0}+\sum_{i: 2 \leqslant 2 i \leqslant k+1} r\left(z_{2 i}, h_{2 i}\right)
\end{gathered}
$$

(т.е. мера $\pi_{n, 1}$ сосредоточена в точках $0, \pm z_{1}, \pm z_{3}, \ldots$, а мера $\pi_{n, 0}$ - в точках $0, \pm z_{2}, \pm z_{4}, \ldots$ и меры симметричны). Положим $h=h_{k+1}=$ const $\in(0,1)$.

Зафиксировав на время наборы $\left\{z_{i}\right\}$, выберем наборы $\bar{h}=\left\{h_{i}, i=1, \ldots, k\right\}$ так, чтобы аннулировать первые $k$ слагаемых в ряде (2.8), т.е. чтобы

$$
\mu_{2 j}\left(\pi_{n, 1}\right)=\mu_{2 j}\left(\pi_{n, 0}\right), \quad j=1, \ldots, k .
$$

Это приводит к системе уравнений относительно $\bar{h}$ :

$$
\sum_{i=1}^{k}(-1)^{i-1} h_{i} z_{i}^{2 j}=(-1)^{k+1} h z_{k+1}^{2 j} ; \quad j=1, \ldots, k .
$$

Положим

$$
Q_{i}(z)=\frac{z^{2}}{z_{i}^{2}} \prod_{1 \leqslant s \leqslant k, s \neq i} \frac{z^{2}-z_{s}^{2}}{z_{i}^{2}-z_{s}^{2}}
$$


Лемма 2.3. При $z_{1}>z_{2}>\cdots>z_{k+1}>0$ наборы $h_{i}=(-1)^{k-i} h Q_{i}\left(z_{k+1}\right)$, $i=1, \ldots, k$, суть решения системь $(2.10)$ u $h_{i}>0, i=1, \ldots, k$.

Д ок а за т ел в с т в о. При $0<z<\min _{1 \leqslant i \leqslant k} z_{i}$ легко непосредственно проверить неравенство $(-1)^{k-i} h Q_{i}(z)>0, i=1, \ldots, k$. Для проверки того, что величины $h_{i}$ удовлетворяют системе (2.10), рассмотрим функции

$$
P_{j}\left(z^{2}\right)=\sum_{i=1}^{k}(-1)^{k-1} h Q_{i}(z) z_{i}^{2 j}+(-1)^{k} h z^{2 j}, \quad j=1, \ldots, k
$$

как полиномы степени $k$ от $x=z^{2}$. Полагая $z_{0}=0$, легко проверить, что $Q_{j}\left(z_{i}\right)$ равно 1 при $i=j$ и 0 при $i \neq j, Q_{j}\left(z_{0}\right)=0 ; i, j=1, \ldots, k$. Отсюда следует, что $P_{j}\left(z_{i}^{2}\right)=0$ при $i=0, \ldots, k$, тах что $P_{j}(x)=0$ тождественно при $j=1, \ldots, k$. В частности, $P_{j}\left(z_{k+1}^{2}\right)=0, j=1, \ldots, k$, что равносильно утверждению леммы.

Вернемся х выбору наборов $\left\{z_{i}\right\}$. Зададимся положительными последовательностями $x=x_{n}=o(1), y=y_{n}=o(1), b=b_{n}=o(1)$ и положим $z_{1}=b ; z_{i}^{2}=b^{2} x y^{i-2}$, $2 \leqslant i \leqslant k+1$. При этом из леммы 2.3 видим, что

$$
h_{1}=h x y^{k-1} \frac{\left(x-x y^{k-1}\right)\left(x y-x y^{k-1}\right) \cdots\left(x y^{k-2}-x y^{k-1}\right)}{(1-x)(1-x y) \cdots\left(1-x y^{k-2}\right)} \sim h x^{k} y^{\phi_{1}(k)}
$$

а при $2 \leqslant i \leqslant k$

$$
\begin{aligned}
h_{i} & =h \frac{x y^{k-1}\left(1-x y^{k-1}\right)\left(x-x y^{k-1}\right) \cdots\left(x y^{i-3}-x y^{k-1}\right)\left(x y^{i-1}-x y^{k-1}\right) \cdots}{x y^{i-2}\left(1-x y^{i-2}\right)\left(x-x y^{i-2}\right) \cdots\left(x y^{i-3}-x y^{i-2}\right)\left(x y^{i-2}-x y^{i-1}\right) \cdots} \\
& \sim h y^{\phi_{i}(k)}
\end{aligned}
$$

где

$$
\phi_{i}(k)= \begin{cases}\frac{k(k-1)}{2} & \text { при } i=1, \\ \frac{k(k-1)-(i-2)(2 k-i+1)}{2} & \text { при } 2 \leqslant i \leqslant k .\end{cases}
$$

Отметим, что $\phi_{i}(k)>0$ при $1 \leqslant i \leqslant k$, и положим $\phi_{1}(k)=\phi_{2}(k)=\phi(k), z=z_{n}=$ $y^{\phi(k)}$.

В силу (2.11), (2.12) при больших $n$ выполнено условие $H_{1}+H_{0} \sim h<1$, так что $\pi_{n, 1}$ - вероятностные меры и $\left|\pi_{n, 1}\right|=b=o(1),\left|\pi_{n, 0}\right|=b x^{1 / 2}=o(1)$. Также кспользуя соотношения $(2.11),(2.12)$, имеем:

$$
\begin{aligned}
& \mathbf{E}_{\pi_{n, 1}}|t|^{p}= \sum_{j: 1 \leqslant 2 j+1 \leqslant k+1} h_{2 j+1} z_{2 j+1}^{p} \sim h b^{p}\left(x^{k} y^{\phi_{1}(k)}+x^{p / 2} y^{\psi_{3}(k, p)}+\cdots\right. \\
&\left.+x^{p / 2} y^{\psi_{2 j+1}(k, p)}+\cdots\right) \sim h b^{p} x^{k} z ; \\
& \mathbf{E}_{\pi_{n}, 0}|t|^{p}= \sum_{j: 2 \leqslant 2 j \leqslant k+1} h_{2 j} z_{2 j}^{p} \sim h b^{p}\left(x^{p / 2} y^{\psi_{2}(k, p)}+\cdots+x^{p / 2} y^{\psi_{2}(k, p)}+\cdots\right) \\
& \sim h b^{p} x^{p / 2} z ; \quad \psi_{i}(k, p)=\phi_{i}(k)+\frac{p(i-2)}{2}
\end{aligned}
$$

поскольку $\psi_{i}(k, p)=\phi(k)+(p-2 k+i-1)(i-2) / 2>\phi(k)$ при $2 k \leqslant p, 2 \leqslant i \leqslant k$, а степень $y$ в последнем члене, входящем в одну из сумм, есть $\psi_{k+1}(k, p)=(k-1) p / 2>\phi(k)$. Аналогичные оценки показывают, что

$$
\begin{aligned}
& \mathbf{E}_{\pi_{n, 1}}|t|^{2(k+1)}=O\left(b^{2(k+1)} x^{k} z\right) \\
& \mathbf{E}_{\pi_{n, 0}}|t|^{2(k+1)}=O\left(b^{2(k+1)} x^{k+1} z\right) .
\end{aligned}
$$

Пусть $R_{n, 0}=U_{n}^{1 / p} R_{n, 0}^{*}, U_{n} \rightarrow \infty$, причем $U_{n}=o\left(n^{a}\right)$ для достаточно малого $a>0$. Выберем $x=x_{n}=o(1), b=b_{n}=o(1), y=y_{n}=o(1)$ так, чтобы

$$
\begin{gathered}
2 h n x^{p / 2} b^{p} z=R_{n, 0}^{p}, \quad n x^{k} b^{p} z \gg\left(R_{n, 1}^{*}\right)^{p}=n^{1 / 2}, \\
x^{k} b^{2(k+1)} z=o\left(n^{-1 / 2}\right) .
\end{gathered}
$$


Полагая $R_{n, 1}^{p}=n h x^{k} b^{p} z / 2$ и используя (2.13)-(2.15), видим, что

$$
\begin{aligned}
& n \mathbf{E}_{\pi_{n, 1}}|t|^{p} \geqslant 2 R_{n, 1}^{p}(1+o(1)), \quad 2 n \mathbf{E}_{\pi_{n, 0}}|t|^{p} \leqslant R_{n, 0}^{p}(1+o(1)), \\
& n\left(\mathbf{E}_{\pi_{n, 1}}|t|^{2(k+1)}\right)^{2}=O\left(n x^{2 k} b^{4(k+1)} z^{2}\right)=o(1) \\
& n\left(\mathbf{E}_{\pi_{n, 0}}|t|^{2(k+1)}\right)^{2}=O\left(n x^{2(k+1)} b^{4(k+1)} z^{2}\right)=o\left(x^{2}\right)=o(1),
\end{aligned}
$$

и в силу леммы 2.2 последовательность $R_{n, 1} \gg R_{n, 1}^{*}$ удовлетворяет условиям п. 1) теоремы 1.1 .

Осталось показать возможность выбора величин, удовлетворяющих (2.16). Положим $b=U_{n}^{-\alpha}, x=n^{-1 / 2 k} U_{n}^{\beta}, 2 h z=U_{n}^{-\gamma} ;$ достаточно выбрать такие $\alpha>0, \beta>0$, $\gamma>0$, чтобы выполнялись соотношения:

$$
\beta=2 \alpha+\frac{2(1+\gamma)}{p}, \quad \gamma+p \alpha<k \beta<\gamma+2(k+1) \alpha .
$$

Чтобы удовлетворить этим соотношениям, достаточно выбрать $\gamma$ так, чтобы $0<\gamma<$ $2 k /(p-2 k$ ) (это обеспечивает неравенство $\delta=2 k(1+\gamma) / p-\gamma>0$ ), и величину $\alpha-$ так, чтобы $\delta / 2<\alpha<\delta /(p-2 k)$. Это возможно при $2 k \leqslant p<2(k+1)$.

Таким образом, п. 1) теоремы 1.1 доказан.

3 а м е ч а н и е 2.2. Фактически мы доказали следующее. Пусть $R_{n, 0}=$ $U_{n} R_{n, 0, p}^{*}, U_{n} \rightarrow \infty, U_{n}=o\left(n^{a}\right)$ для некоторого $0<a \leqslant a(p)$. Тогда существует такое $\delta(p)>0$, что $\gamma_{n} \rightarrow 1$ при $R_{n, 1}=O\left(U_{n}^{\delta} R_{n, 1, p}^{*}\right), 0<\delta \leqslant \delta(p)$.

Кроме того, с учетом замечания 2.1, в теореме 1.1 множества $V_{n, l}$ можно заменить кольцами $\left\{c_{l} \leqslant\|v\|_{p} / R_{n, l} \leqslant C_{l}\right\}$ при любых $0<c_{l}<C_{l}, l=0,1$.

\section{3. Верхние границы в задаче 1}

3.1. Метод построения верхних границ. Пусть $R_{n, 0}=o\left(R_{n, 0}^{*}\right) ; R_{n, 1}=$ $U_{n} R_{n, 1}^{*}, U_{n} \rightarrow \infty$. Мы покажем, что в условиях п. 2) теоремы 1.1 состоятельными в минимаксном смысле являются тесты $\psi_{n, p}(x)=1_{\left\{L_{n, p}(x)>T_{n}\right\}}$, основанные на статистиках $L_{n}=L_{n, p}(x)=n^{-1 / 2} \sum_{i=1}^{n} u_{p}\left(x_{i}\right)$ и носледовательности $T_{n}=U_{n}^{p / 2} \rightarrow \infty$ (вид функций $u_{p}(x)$ мы уточним ниже).

Для оценки вероятностей ошибок I рода $\alpha\left(\psi_{n, p}, v\right)=\mathbf{P}_{n, v}\left\{L_{n}>T_{n}\right\}, v \in V_{n, 0}$, при $T_{n}>\mathbf{E}_{n, v}\left(L_{n}\right)$ используем неравенство Чебышева:

$$
\mathbf{P}_{n, v}\left\{L_{n}>T_{n}\right\}=\mathbf{P}_{n, v}\left\{L_{n}-\mathbf{E}_{n, v}\left(L_{n}\right)>T_{n}-\mathbf{E}_{n, v}\left(L_{n}\right)\right\} \leqslant \frac{\mathbf{D}_{n, v}\left(L_{n}\right)}{\left(T_{n}-\mathbf{E}_{n, v}\left(L_{n}\right)\right)^{2}},
$$

так что достаточно показать, что

$$
\sup _{v \in V_{n, 0}} \mathbf{D}_{n, v}\left(L_{n}\right)=O(1), \quad \sup _{v \in V_{n, 0}} \mathbf{E}_{n, v}\left(L_{n}\right)=o(1) .
$$

Оценим вероятности ошибок II рода $\beta\left(\psi_{n, p}, v\right)=\mathbf{P}_{n, v}\left\{L_{n} \leqslant T_{n}\right\}, v \in V_{n, 1}$. Также используя неравенство Чебышева при $T_{n}<\mathbf{E}_{n, v}\left(L_{n}\right)$, имеем

$$
\mathbf{P}_{n, v}\left\{L_{n} \leqslant T_{n}\right\}=\mathbf{P}_{n, v}\left\{\mathbf{E}_{n, v}\left(L_{n}\right)-L_{n} \geqslant \mathbf{E}_{n, v}\left(L_{n}\right)-T_{n}\right\} \leqslant \frac{\mathbf{D}_{n, v}\left(L_{n}\right)}{\left(\mathbf{E}_{n, v}\left(L_{n}\right)-T_{n}\right)^{2}},
$$

так что достаточно показать, что

$$
\inf _{v \in V_{n, 1}} \frac{\mathbf{E}_{n, v}\left(L_{n}\right)}{T_{n}} \rightarrow \infty, \quad \sup _{v \in V_{n, 1}} \frac{\mathbf{D}_{n, v}\left(L_{n}\right)}{\mathbf{E}_{n, v}^{2}\left(L_{n}\right)}=o(1) .
$$

Ниже мы будем использовать неравенства: при $0 \leqslant a<b$

$$
n^{-1} \sum_{i=1}^{n}\left|x_{i}\right|^{a} \leqslant\left(n^{-1} \sum_{i=1}^{n}\left|x_{i}\right|^{b}\right)^{a / b}, \quad\left(\sum_{i=1}^{n}\left|x_{i}\right|^{b}\right)^{a / b} \leqslant \sum_{i=1}^{n}\left|x_{i}\right|^{a} .
$$


3.2. Случай $p \leqslant 2$. Положим $u_{p}(x)=|x|^{p}-c(p)$, где $c(p)=\mathbf{E}|\xi|^{p}, \xi$ есть стандартная гауссовская случайная величина.

Лемма 3.1. Пусть $p \leqslant 2, h_{p}(t)=\mathbf{E} u_{p}(\xi+t), H_{p}(t)=\mathbf{D} u_{p}(\xi+t), x_{+}=\max (x, 0)$. Cуиествуют тахие постовнные $C_{i}=C_{i}(p)>0$, ито для любого $t \in \mathbf{R}^{1}$

$$
C_{1} \min \left(t^{2},|t|^{p}\right) \leqslant h_{p}(t) \leqslant C_{2}|t|^{p} ; \quad H_{p}(t) \leqslant C_{3}\left(1+|t|^{\left.2(p-1)_{+}\right) .}\right.
$$

Д ок а з а т ель с т в о. Нетрудно видеть, что $h_{p}(t)$ - четная бесконечно дифференцируемая функция, строго возрастающая по $|t|, h_{p}(0)=0, h_{p}^{\prime \prime}(0)>0$. Поэтому для любого $b>0$ найдутся такие постоянные $B_{i}=B_{i}(p, b)>0$, что

$$
B_{1} t^{2} \leqslant h_{p}(t) \leqslant B_{2} t^{2}, \quad|t| \leqslant b .
$$

С другой стороны, используя неравенство

$$
|a+b|^{p} \leqslant A(p)\left(|a|^{p}+|b|^{p}\right), \quad A(p)=2^{(p-1)}+,
$$

получим оценки

$$
-2 c(p)+\frac{|t|^{p}}{A(p)} \leqslant h_{p}(t) \leqslant(A(p)-1) c(p)+A(p)|t|^{p} .
$$

Из (3.4), (3.6) вытекают требуемые оценки для $h_{p}(t)$. Далее, используя при $p>1$ формулу Тейлора:

$$
|| t+\left.\xi\right|^{p}-|t|^{p}|=p| \theta \xi+\left.t\right|^{p-1}|\xi| \leqslant p\left(|\xi|^{p-1}+|t|^{p-1}\right)|\xi|
$$

а при $0<p \leqslant 1$ - вытекаюощю из (3.5) оценку ||$t+\left.\xi\right|^{p}-\left.|t|^{p}|\leqslant| \xi\right|^{p}$, с учетом неравенства $\mathbf{D}\left(|t+\xi|^{p}\right) \leqslant \mathbf{E}\left(|t+\xi|^{p}-|t|^{p}\right)^{2}$, получим требуемые оценки для $H_{p}(t)$. Лемма доказана.

Для оценки вероятностей ошибок I рода проверим выполнение соотношений (3.1). Используя лемму 3.1, имеем при $R_{n, 0} \ll R_{n, 0}^{*}=n^{1 / 2 p}$ :

$$
\begin{aligned}
\mathbf{E}_{n, v}\left(L_{n}\right) & =n^{-1 / 2} \sum_{i=1}^{n} h_{p}\left(v_{i}\right) \leqslant C_{2} n^{-1 / 2} \sum_{i=1}^{n}\left|v_{i}\right|^{p} \leqslant C_{2} n^{-1 / 2} R_{n, 0}^{p}=o(1), \\
\mathbf{D}_{n, v}\left(L_{n}\right) & =n^{-1} \sum_{i=1}^{n} H_{p}\left(v_{i}\right) \leqslant C_{3}\left(1+n^{-1} \sum_{i=1}^{n}\left|v_{i}\right|^{2(p-1)_{+}}\right) \\
& \leqslant C_{3}\left(1+D_{n}^{2(p-1)_{+} / p}\right)=O(1), \quad D_{n}=n^{-1} \sum_{i=1}^{n}\left|v_{i}\right|^{p}
\end{aligned}
$$

(мы использовали также первое неравенство (3.3), поскольку $2(p-1)_{+} \leqslant p$ при $0<p \leqslant 2$, и соотношения $\left.D_{n} \leqslant n^{-1} R_{n, 0}^{p}=o(1)\right)$.

Для оценки вероятностей ошибок II рода проверим выполнение соотношений (3.2). Также используя лемму 3.1 , имеем

$$
\mathbb{E}_{n, v}\left(L_{n}\right) \geqslant C_{1} n^{-1 / 2}\left(\sum_{i \in I} v_{i}^{2}+\sum_{i \notin I}\left|v_{i}\right|^{p}\right), \quad I=I_{n}(v)=\left\{i:\left|v_{i}\right| \leqslant 1\right\} .
$$

Поскольку

$$
S_{n, 1}+S_{n, 2}=\sum_{i \in I}\left|v_{i}\right|^{p}+\sum_{i \notin I}\left|v_{i}\right|^{p} \geqslant R_{n, 1}^{p}
$$

при $v \in V_{n, 1}$, то одна из сумм $S_{n, 1}, S_{n, 2}$ не меньше $R_{n, 1}^{p} / 2$. Если это первая, то, используя первое неравенство (3.3), имеем

$$
n^{-1 / 2} \sum_{i \in I} v_{i}^{2} \geqslant n^{1 / 2-2 / p} S_{n, 1}^{2 / p} \geqslant n^{1 / 2-2 / p}\left(\frac{R_{n, 1}^{p}}{2}\right)^{2 / p} \asymp U_{n}^{2}
$$

если же это вторая, то

$$
n^{-1 / 2} S_{n, 2} \geqslant n^{-1 / 2}\left(\frac{R_{n, 1}}{2}\right)^{p} \asymp U_{n}^{p} n^{(2-p) / 4} .
$$


В любом случае выполнено первое из соотношений (3.2).

Далее, из оценох дисперсий, приведенных в (3.8), имеем: $\mathbf{D}_{n, v}\left(L_{n}\right)=O(1)$ при $p \leqslant 1$ или $D_{n}=O(1)$; если же $1 \leqslant p \leqslant 2$ и $D_{n}=D_{n, 1}+D_{n, 2} \rightarrow \infty$, то, используя неравенство (3.5), получим:

$$
\begin{gathered}
\mathbf{D}_{n, v}\left(L_{n}\right)=O\left(D_{n, 1}^{2(p-1) / p}+D_{n, 2}^{2(p-1) / p}\right) \\
D_{n, 1}=n^{-1} \sum_{i \in I}\left|v_{i}\right|^{p}, \quad D_{n, 2}=n^{-1} \sum_{i \notin I}\left|v_{i}\right|^{p} .
\end{gathered}
$$

При этом, если $D_{n, 1} \rightarrow \infty$, то, используя первое неравенство (3.3), имеем:

$$
D_{n, 1}^{2(p-1) / p} \leqslant\left(n^{-1} \sum_{i \in I} v_{i}^{2}\right)^{p-1} \leqslant n^{-1} \sum_{i \in I}\left|v_{i}\right|^{2}=o\left(\mathbf{E}_{n, v}\left(L_{n}\right)\right)
$$

так как $p-1 \leqslant 1$; если $D_{n, 2} \rightarrow \infty$, то

$$
D_{n, 2}^{2(p-1) / p} \leqslant D_{n, 2} \ll n^{-1 / 2} \sum_{i \notin I}\left|v_{i}\right|^{p} \leqslant \frac{\mathbf{E}_{n, v}\left(L_{n}\right)}{C_{1}},
$$

так как $2(p-1) / p \leqslant 1$. В любом случае выполнено второе из соотношений (3.2). Таким образом, п. 2) теоремы 1.1 при $p \leqslant 2$ доказан.

3.3. Случай $p \geqslant 2$. Пусть $2 k \leqslant p<2(k+1), k \geqslant 1$ и целое. Пусть $\xi$ есть стандартная гауссовская случайная величина, $f_{p}(t)=\mathbf{E}|\xi+t|^{p}-$ четная бесконечно дифференцируемая функция. Пусть $c_{j}(p)=f_{p}^{(2 j)}(0)-$ производные этой функции в точке 0. Рассмотрим такие функцик $\phi_{j}(x)$, что $\mathbf{E} \phi_{j}(\xi+t)=t^{2 j}, j=0,1, \ldots, k-1$ (это полиномы степени $2 j$; их явный вид $\phi_{j}(x)=\mathbf{E}(x+i \xi)^{2 j}, i=\sqrt{-1}$, см. [12], [13]). Положим

$$
u_{p}(x)=|x|^{p}-\sum_{j=0}^{k-1} \frac{c_{j}(p) \phi_{j}(x)}{(2 j) !}=|x|^{p}-Q_{k-1}\left(x^{2}\right),
$$

$Q_{k-1}-$ полином степени $k-1$.

Лемма 3.2. Пусть $p \geqslant 2, h_{p}(t)=\mathbf{E} u_{p}(\xi+t)=f_{p}(t)-P_{k-1}\left(t^{2}\right), P_{k-1}$ - полином степени $k-1 ; H_{p}(t)=\mathbf{D} u_{p}(\xi+t)$. Сучествуют такие постоянявзе $C_{i}=C_{i}(p)>0$, uто для мобого $t \in \mathbf{R}^{1}$

$$
C_{1}|t|^{p} \leqslant h_{p}(t) \leqslant C_{2}\left(|t|^{p}+t^{2 k}\right) ; \quad H_{p}(t) \leqslant C_{3}\left(1+|t|^{2(p-1)}\right) .
$$

Доказате льство. Нетрудно видеть, что $h_{p}(t)$ - четная бесконечно дифференцируемая функция, $h_{p}^{(j)}(0)=0, j=0,1, \ldots, 2 k-1 ; h_{p}^{(2 k)}(0)>0$. Разла$\operatorname{ras}|\xi+t|^{p}$ по формуле Тейлора в точке $\xi$ и усредняя, получим

$$
h_{p}(t)=d_{k} \mathbf{E}\left(|\xi+\theta t|^{p-2 k}\right) t^{2 k}, \quad \theta=\theta(t, \xi) \in[0,1],
$$

где $d_{k}=p(p-1) \cdots(p-2 k+1) /(2 k)$ ! $>0$. Поскольку среднее в (3.9) ограничено и отделено от 0 на любом конечном интервале по $t$, для любого $b>0$ найдутся такие постоянные $B_{i}=B_{i}(p, b)>0$, что

$$
B_{0}|t|^{p} \leqslant B_{1} t^{2 k} \leqslant h_{p}(t) \leqslant B_{2} t^{2 k}, \quad|t| \leqslant b .
$$

Используя неравенство (3.5), аналогично (3.6) получаем:

$$
\mathbf{E}\left(|\xi+\theta t|^{p-2 k}\right) \leqslant A(p-2 k)\left(\mathbf{E}|\xi|^{p-2 k}+|t|^{p-2 k}\right) .
$$

Из (3.9)-(3.11) вытекают верхние границы для $h_{p}(t)$.

Снова используя неравенство (3.5), получим: $|\xi+t|^{p} \geqslant|t|^{p} / A(p)-|\xi|^{p}$, откуда следует, что для достаточно большого $b>0$ и некоторого $B>0$

$$
h_{p}(t) \geqslant \frac{|t|^{p}}{A(p)}-\left|P_{k-1}\left(t^{2}\right)\right|-\mathbf{E}|\xi|^{p} \geqslant B|t|^{p} \quad \text { при }|t|>b .
$$

Таким образом получаем нижние границы для $h_{p}(t)$. 
Используя неравенство, аналогичное (3.7), с учетом неравенств

$$
\mathbf{D} u_{p}(\xi+t)=\mathbf{D}\left(u_{p}(\xi+t)-|t|^{p}\right) \leqslant 2 \mathbf{E}\left(|t+\xi|^{p}-|t|^{p}\right)^{2}+2 \mathbf{E} Q_{k-1}^{2}\left((\xi+t)^{2}\right),
$$

$\mathbf{E} Q_{k-1}^{2}\left((\xi+t)^{2}\right)=O\left(t^{4(k-1)}\right)$, получим требуемые оценки для $H_{p}(t)$. Лемма доказана.

Для оценки вероятностей ошибок I рода проверим выполнение соотношений (3.1). Используя лемму 3.2, имеем:

$$
\mathbf{E}_{n, v}\left(L_{n}\right)=n^{-1 / 2} \sum_{i=1}^{n} h_{p}\left(v_{i}\right) \leqslant C_{2} n^{-1 / 2}\left(S_{1}+S_{2}\right),
$$

где в силу первого неравенства (3.3)

$$
S_{1}=\sum_{i=1}^{n}\left|v_{i}\right|^{p} \leqslant R_{n, 0}^{p}, \quad S_{2}=\sum_{i=1}^{n} v_{i}^{2 k} \leqslant n^{1-2 k / p} S_{1}^{2 k / p},
$$

так что при $R_{n, 0} \ll R_{n, 0}^{*}=n^{1 / p-1 / 4 k}$

$$
\sup _{v \in V_{n, 0}} \mathbf{E}_{n, v}\left(L_{n}\right) \leqslant n^{-1 / 2}\left(R_{n, 0}^{p}+n^{1-2 k / p} R_{n, 0}^{2 k}\right)=o\left(n^{(2 k-p) / 4 k}+1\right)=o(1) .
$$

Далее, используя второе неравенство (3.3) и неравенство $2(p-1) \geqslant p$, имеем:

$$
\begin{aligned}
\mathbf{D}_{n, v}\left(L_{n}\right) & =n^{-1} \sum_{i=1}^{n} H_{p}\left(v_{i}\right) \leqslant C_{3}\left(1+n^{-1} \sum_{i=1}^{n}\left|v_{i}\right|^{2(p-1)}\right) \\
& \leqslant C_{3}\left(1+n^{-1} S_{1}^{2(p-1) / p}\right)=O\left(1+n^{r}\right)=O(1),
\end{aligned}
$$

так как легко проверить, что $r=1-2 / p-(p-1) / 2 k<0$ при $p \geqslant 2 k>1$.

Для оценки вероятностей ошибок II рода проверим выполнение соотношений (3.2). Также используя лемму 3.2 , имеем

$$
\mathbf{E}_{n, v}\left(L_{n}\right) \geqslant C_{1} n^{-1 / 2} S_{1} \geqslant C_{1} n^{-1 / 2} R_{n, 1}^{p} \asymp U_{n}^{p},
$$

так что справедливо первое из соотношений (3.2). Из (3.12), (3.13) имеем: $\mathbf{D}_{n, v}\left(L_{n}\right) \leqslant$ $B n^{-1 / p}\left(\mathbf{E}_{n, v} L_{n}\right)^{2(p-1) / p}=o\left(\left(\mathbf{E}_{n, v} L_{n}\right)^{2}\right)$, так что справедливо и второе из соотношений (3.2). Это завершает доказательство теоремы 1.1 .

\section{4. Доказательство теоремы 1.2}

4.1. Нижние границы. Пусть $\varphi(t)$ есть [ $\sigma+2]$-непрерывно дифференцируемая функция на $\mathbf{R}^{1}$ с носителем в интервале $(0,1)$ и такая, что $\|\varphi\|_{2}=1$. Тогда для любого целого $n>0$ набор функций $\varphi_{i, n}(t)=n^{1 / 2} \varphi(n t-i+1), i=1, \ldots, n$, можно рассматривать как ортонормальную систему функций в $L_{2}(0,1)$ с непересекающимися носителями, и найдутся такие не зависящие от $n$ постоянные $C_{1} \geqslant C_{0}>0$, что при всех $p \geqslant 1, h \geqslant 1, n>0$ справедливы неравенства:

$$
C_{0}\left\|g_{\theta, n}\right\|_{\sigma, p, h} \leqslant\|\theta\|_{p} n^{\sigma+1 / 2-1 / p} \leqslant C_{1}\left\|g_{\theta, n}\right\|_{\sigma, p, h} ; \quad \theta \in \mathbf{R}^{n},
$$

где $g_{\theta, n}=\sum_{i=1}^{n} \theta_{i} \varphi_{i, n},\|\theta\|_{p}=\left(\sum_{i=1}^{n}\left|\theta_{i}\right|^{p}\right)^{1 / p} ;$ аналогичные неравенства при $\sigma=0$ справедливы для $L_{p}$-нормы:

$$
C_{0}\left\|g_{\theta, n}\right\|_{p} \leqslant\|\theta\|_{p} n^{1 / 2-1 / p} \leqslant C_{1}\left\|g_{\theta, n}\right\|_{p}, \quad \theta \in \mathbf{R}^{n} .
$$

Обозначим $\phi_{l}(p)$ показатель степени $n$ в $R_{n, l, p}^{*}: R_{n, l, p}^{*}=n^{\phi_{l}(p)}$, и пусть $\psi_{l}(p)=$ $\frac{1}{2}-1 / p+\phi_{l}(p)$. Отметим, что $\psi_{l}(p)>0, l=0,1$. Положим $n_{\varepsilon}^{*}=n_{*}=\varepsilon^{-1 /\left(\sigma+\psi_{1}(p)\right)}$, т.e. $\epsilon n_{*}^{\sigma+1 / 2-1 / p} R_{n_{*}, 1, p}^{*}=1$. При этом

$$
\begin{aligned}
& \rho_{\varepsilon, 1, p}^{*}=\varepsilon n_{*}^{1 / 2-1 / p} R_{n_{*}, 1, p}^{*}=\varepsilon n_{*}^{\psi_{1}(p)}, \\
& \rho_{\varepsilon, 0, p}^{*}=\varepsilon n_{*}^{1 / 2-1 / p} R_{n_{*}, 0, p}^{*}=\varepsilon n_{*}^{\psi_{0}(p)} .
\end{aligned}
$$


Пусть $\rho_{\varepsilon, 0}=W_{\varepsilon} \rho_{\varepsilon, 0, p}^{*}, W_{\varepsilon} \rightarrow \infty, W_{\varepsilon}=o\left(n_{*}^{b}\right)$ для малого $b>0$. Положим $n=n_{\varepsilon} \asymp$ $W_{\varepsilon}^{-\alpha} n_{*} \rightarrow \infty, \alpha>0, U_{\varepsilon}=W_{\varepsilon}^{\beta}, \beta=1+\alpha \psi_{0}(p)>0$. Имеем:

$$
\rho_{\varepsilon, 0} \asymp U_{\varepsilon} \varepsilon n^{1 / 2-1 / p} R_{n, 0, p}^{*} \text {. }
$$

Выбирая $\gamma: 0<\gamma<\min \left(\beta \delta(p)-\alpha \psi_{1}(p), \sigma \alpha\right)$, где $\delta(p)$ есть величина из замечания 2.2 (это возможно при малом $\alpha>0$ ), положим

При этом

$$
\rho_{\varepsilon, 1}=W_{\varepsilon}^{\gamma} \rho_{\varepsilon, 1, p}^{*}=U_{e}^{\delta} \varepsilon n^{1 / 2-1 / p} R_{n, 1, p}^{*}, \quad 0<\delta<\delta(p) .
$$

$$
\varepsilon U_{\varepsilon}^{\delta} R_{n, 1, p}^{*} n^{\sigma+1 / 2-1 / p}=W^{\gamma}\left(\frac{n}{n_{*}}\right)^{\sigma} \asymp W^{\gamma-\sigma \alpha}=o(1) .
$$

Покажем, что семейство $\rho_{\varepsilon, 1}$ удовлетворяет условиям п. 1) теоремы 1.2. Обозначим $G_{n}$ линейную оболочку системы $\left\{\varphi_{i, n} ; i=1, \ldots, n\right\}$. Положим $x=\left(x_{1}, \ldots, x_{n}\right)$, $v=v(f)=\left(v_{1}, \ldots, v_{n}\right)$,

$$
\begin{gathered}
x_{i}=\varepsilon^{-1} \int \varphi_{i, n}(t) d X_{\varepsilon}(t), \quad v_{i}=\varepsilon^{-1}\left(f, \varphi_{i, n}\right) \\
V_{n, l}=\left\{v(f): f \in G_{n} \cap F_{\varepsilon, l}\right\}, \quad l=0,1
\end{gathered}
$$

$\mathbf{P}_{\varepsilon, f}$-распределения случайных векторов $\boldsymbol{x}$ есть $\mathbf{P}_{n, v}$-распределения на $\left(\mathbf{R}^{n}, \mathscr{B}^{n}\right)$. В силу неравенств (4.1), (4.2) и соотношений (4.3)-(4.6) множества $V_{n, l}$ содержат кольща $\left\{c_{l} \leqslant\|v\|_{p} / R_{n, l} \leqslant C_{l}\right\}$ при $R_{n, 0}=U_{\varepsilon} R_{n, 0, p}^{*}, R_{n, 1}=U_{\varepsilon}^{\delta} R_{n, 1, p}^{*}$ и некоторых $0<c_{l}<$ $C_{l}, l=0,1$. Поэтому утверждение п. 1) теоремы 1.2 вытекает непосредственно из замечания 2.2 .

4.2. Верхние границы. Рассмотрим целочисленное семейство $n=n_{\varepsilon} \asymp n_{*}$, где $n_{*}$ - величины, определенные в п. 4.1. Пусть

$$
\begin{aligned}
& \rho_{\varepsilon, 0}=\varepsilon n^{1 / 2-1 / p} R_{n, 0} \ll \rho_{\varepsilon, 0}^{*} \asymp \varepsilon n^{1 / 2-1 / p} R_{n, 0, p}^{*}, \\
& \rho_{\varepsilon, 1}=\varepsilon n^{1 / 2-1 / p} R_{n, 1} \gg \rho_{\varepsilon, 1}^{*} \asymp \varepsilon n^{1 / 2-1 / p} R_{n, 1, p}^{*} ;
\end{aligned}
$$

при этом $\rho_{\varepsilon, 1}^{*} \asymp n^{-\sigma}$ в силу первого соотношения (4.3) и определения $n_{*}$. Пусть $\Delta_{n}$ - равномерное разбиение $[0,1]$ на $n$ интервалов $\delta_{1, n}, \ldots, \delta_{n, n}, \phi_{i, n}=n^{1 / 2} 1_{\delta_{i, n}}$ ортонормальная система в $L_{2}(0,1), \operatorname{Pr}_{n}$ есть оператор ортонормального проектирования в $L_{2}(0,1)$ на пространст»о функций, постоянных на интервалах разбиения $\Delta_{n}: \operatorname{Pr}_{n}(f)=\sum_{i=1}^{n}\left(f, \phi_{i, n}\right) \phi_{i, n}$. Положим $x=x\left(X_{e}\right)=\left(x_{1}, \ldots, x_{n}\right), v=v(f)=$ $\left(v_{1}, \ldots, v_{n}\right)$,

$$
\begin{gathered}
x_{i}=\varepsilon^{-1} \int \phi_{i, \pi}(t) d X_{\varepsilon}(t), \quad v_{i}=\varepsilon^{-1}\left(f, \phi_{i, n}\right) ; \\
V_{n, l}=\left\{v(f): f \in F_{\varepsilon, l}\right\}, \quad l=0,1 .
\end{gathered}
$$

Ясно, что $\mathbf{P}_{\varepsilon, f}$-распределение векторов $x$ есть $\mathbf{P}_{n, v}$-распределение на $\left(\mathbf{R}^{n}, \mathscr{B}^{n}\right)$. Легко видеть, что

$$
\varepsilon n^{1 / 2-1 / p}\|v(f)\|_{p}=\left\|\operatorname{Pr}_{n}(f)\right\|_{p} \leqslant\|f\|_{p}
$$

и в силу (4.9) множество $V_{n, 0}$ леж ит в $l_{p}^{n}$-шаре радиуса $R_{n, 0} \ll R_{n, 0, p}^{*}$.

Воспользуемся следующим неравенством. Пусть $\|f\|_{\sigma, p, \infty} \leqslant C$ (это случай наиболее «широкой» альтернативы). Тогда найдутся такие не зависящие от $\boldsymbol{n}$ положительные постоянные $B_{1}, B_{2}$, что

$$
\|\operatorname{Pr} n\|_{p} \geqslant B_{1}\|f\|_{p}-B_{2} n^{-\sigma} \text {. }
$$

Неравенство (4.10) дохазано в [3 (см. неравенство (5.16)) для шаров Никольского $H_{p}^{\sigma}$, совпадающих с шарами Бесіва при $\boldsymbol{h}=\infty$. Доказательство основано на возможности кусочной аппроксимации иункций из шаров Никольского $H_{p}^{\sigma}$ полиномами степени, зависящей от $\sigma$, с точнсстью $n^{-\sigma}$ в $L_{p}$-норме, где $n-$ число интервалов аппроксимации.

Поскольку $\|f\|_{p} \geqslant \rho_{\varepsilon, 1} \gg \rho_{\varepsilon, 1}^{*} \asymp n^{-\sigma}$ при $f \in F_{\varepsilon, 1}$, в силу неравенства (4.10) множество $V_{n, 1}$ лежит вне $l_{p}^{n}$-шара радиуса порядка $R_{n, 1} \gg R_{n, 1, p}^{*}$.

Поэтому требуемое утверждеяие непосредственно следует из п. 2) теоремы 1.1. Состоятельными в минимаксном смысле являются тесты $\psi_{\varepsilon, p}\left(X_{\varepsilon}\right)=\psi_{n, p}\left(x\left(X_{\varepsilon}\right)\right)$, где тесты $\psi_{n, p}(x)$ построены при докизательстве п. 2) теоремы 1.1. 


\section{СПИСОК ЛИТЕРАТУРЫ}

1. Ингстер Ю. И. О минимаксном непараметрическом обнаружении сигнала в гауссовском белом шуме. - Проблемы передачи информации, 1982, т. 18, № 2, с. 6173.

2. Ingster Yu. I. Asymptotically minimax testing of nonparametric hypothesis. - Proceedings of the Fourth Vilnius Conference on Probability Theory and Mathematical Statistics. Vol. 1. Utrecht: VNU Sci. Press, 1987, p. 553-573.

3. Ingster $Y u$. I. Asymptotically minimax hypothesis testing for nonparametric alternatives. I, II, III. - Math. Methods Statist., 1993, v. 2, № 2, p. 85-114; № 3, p. 171-189; № 4, p. 249-268.

4. Суслина И.А. Минимахсное обнаружение сигнала для $l_{q}$-эллипсоидов с удаленным $l_{p}$-шаром. - Зап. науч. семин. ПОМИ, 1993, т. 207, с. 127-137.

5. Ингстер Ю. И. Минимаксное обнаружение сигнала при невырожденных функцияХ потерь и экстремальные выпуклые задачи. - Зап. науч. семин. ПОМИ, 1996, T. 228, c. $162-188$.

6. Суслияа И. А. Экстремальные задачи, возникаюшие при минимаксном обнаружении сигнала для $l_{q}$-эллипсондов с удаленным $l_{p}$-шаром. - Зап. науч. семин. ПОМИ, 1996, т. 228, с. 312-332.

7. Lepski O.V., Spokoiny V. G. Minimax nonparametric hypothesis testing: the case of an inhomogeneous alternative. - Bernoulli, 1999, v. 5, № 2, p. 333-358.

8. Spokoiny V.G. Adaptive and spatially adaptive testing of nonparametric hypothesis. - Math. Methods Statist., 1998, v. 7, № 3, p. 245-273.

9. Ingster Y.I., Suslina I. A. Minimax nonparametric hypothesis testing for ellipsoids and Besov bodies. Report № 12. Berlin: Weierstrass Institute for Applied Analysis and Stochastics, 1997, $88 \mathrm{p}$.

10. Donoho D.L., Johnstone I. M., Kerkyacharian G., Picard D. Wavelet shrinkage: asymptopia? - J. Roy. Statist. Soc., 1995, v. 57, № 2, p. 301-369.

11. Triebel $H$. Theory of Function Spaces. II. Basel: Birkhäuser, 1992, 370 p.

12. Lepski O. V., Nemirovski A., Spokoiny V. G. On estimation of non-smooth functionals. Technical Report № 297. Berlin: Weierstrass Institute for Applied Analysis and Stochastics, 1996.

13. Ибрагимов И. А., Немировский А. С., Хасьминский Р. З. Некоторые задачи непараметрического оценивания в гауссовском белом шуме. - Теория вероятн. и ее примен., 1986, т. XXXI, в. 3, с. 451-466.

Поступила в редакцию 16.III.1998

(C) $2000 \mathrm{r}$.

МАЛЫШЕВ В. А.*

\section{МАКРОРАЗМЕРНОСТЬ - ИНВАРИАНТ ЛОКАЛЬНОЙ ДИНАМИКИ}

На множестве счетных графов с метками определяется марковский процесс. Переходы в нем - локальные преобразования графа. Определяется махроразмерность бесконечного графа и доказывается, что она является инвариантом такой динамики.

Клочевые слова и фразы: марковский процесс, макроразмерность графа, инвариант динамики.

* INRIA, France; e-mail: Vadim.Malyshev@inria.fr 